\title{
Author Correction: The Cardamine enshiensis genome reveals whole genome duplication and insight into selenium hyperaccumulation and tolerance
}

Chuying Huang, Hongqing Yin, Xibiao Yang, Yuan Gao, Tuo Li, Bo Wu, Meng Ren, Zixiong Zhang, Jun Ding, Jianhua Gao, Dan Wen, Xingzhi Ye, Ling Liu, Huan Wang, Guogen Sun, Yi Zou, Nansheng Chen (1) and Li Wang

Correction to: Cell Discovery (2021) 7:62

https://doi.org/10.1038/s41421-021-00286-x

Published online 10 August 2021

In the original publication of this article ${ }^{1}$ one of the author's name was incorrect. In this correction article, the correct and incorrect name are indicated.

Previous incorrect name:

Hongqin Ying

Corrected name:

Hongqing Yin
Previous incorrect name:

These authors contributed equally: Chuying Huang, Hongqin Ying, Xibiao Yang, Li Wang.

Corrected name:

These authors contributed equally: Chuying Huang, Hongqing Yin, Xibiao Yang, Li Wang.

Published online: 23 September 2021

\section{Reference}

1. Huang, C. et al. The Cardamine enshiensis genome reveals whole genome duplication and insight into selenium hyperaccumulation and tolerance. Cell Discov. 7, 62 (2021) 